\title{
REVIEW
}

\section{The influence of drug use on fall incidents among nursing home residents: a systematic review}

\author{
Carolyn S. Sterke, ${ }^{1,4}$ Arianne P. Verhagen, ${ }^{2}$ Ed F. van Beeck ${ }^{3}$ \\ and Tischa J. M. van der Cammen ${ }^{4}$ \\ ${ }^{1}$ De StromenOpmaatGroep, Nursing Home Smeetsland, Rotterdam, the Netherlands \\ ${ }^{2}$ Department of General Practice, Erasmus University Medical Center Rotterdam, the Netherlands \\ ${ }^{3}$ Department of Public Health, Erasmus University Medical Center Rotterdam, the Netherlands \\ ${ }^{4}$ Section of Geriatric Medicine, Department of Internal Medicine, Erasmus University Medical Center Rotterdam, \\ the Netherlands
}

Background: Falls are a major health problem among the elderly, particularly in nursing homes. Abnormalities of balance and gait, psychoactive drug use, and dementia have been shown to contribute to fall risk.

Methods: We conducted a systematic review of the literature to investigate which psychoactive drugs increase fall risk and what is known about the influence of these drugs on gait in nursing home residents with dementia. We included studies with a prospective cohort design on psychoactive drug use in nursing homes with dementia residents and with falls as an outcome measure.

Results: Seventeen studies were included in the review. Pooled risk estimates were not calculated because there was no homogeneity across studies. We assessed the strength of evidence for psychoactive drugs as a prognostic factor for falls by defining four levels of evidence: strong, moderate, limited or inconclusive. Strong evidence was defined as consistent findings $(\geq 80 \%)$ in at least two high quality cohorts. We found strong evidence that the use of multiple drugs (3/3 cohorts, effect sizes 1.30-10.30), antidepressants (10/12 cohorts, effect sizes $1.10-7.60)$, and anti-anxiety drugs ( $2 / 2$ cohorts, effect sizes $1.22-1.32$ ) is associated with increased fall risk. The evidence for the association of other psychoactive drug classes with fall risk was limited or inconclusive.

Conclusions: Research on the contribution of psychoactive drugs to fall risk in nursing home residents with dementia is limited. The scarce evidence shows,

Correspondence should be addressed to: Dr Tischa J. M. van der Cammen, Associate Professor of Geriatric Medicine, Department of Internal Medicine, Erasmus University Medical Center, Room D442, PO Box 2040, NL-3000 CA Rotterdam, The Netherlands. Phone: +31 (0) 10 703.59.79; Fax: +31 (0) 10 703.47.68. Email: t.vandercammen@erasmusmc.nl. Received 11 Dec 2007; revision requested 11 Jan 2008; revised version received 24 Jan 2008; accepted 29 Jan 2008. First published online 17 April 2008. 
however, that multiple drugs, antidepressants and anti-anxiety drugs increase fall risk in nursing home populations with residents with dementia.

Key words: falls, gait, psychoactive drugs, dementia, nursing homes

\section{Introduction}

Falls are a major health problem among the elderly, particularly in nursing homes (Bueno-Cavanillas et al., 2001; Krueger et al., 2001; Jensen et al., 2002; Heinze et al., 2007). Abnormalities of balance and gait (Tinetti et al., 1988; Speechley and Tinetti, 1990; Studenski et al., 1994), psychoactive drug use (Tinetti et al., 1988; Leipzig et al., 1999; Ensrud et al., 2002), and dementia (Morris et al., 1987; van Doorn et al., 2003) have been shown to contribute to fall risk. Gait and balance problems usually occur in the more advanced stages of dementia (Nakamura et al., 1996), and might be due to the use of psychoactive drugs such as antipsychotics, antidepressants and sedatives (Lord et al., 1995).

It is generally known that nursing home residents with dementia have an increased fall risk; however, the additive effect of psychoactive drugs to fall risk in such residents is not known. Also, the mechanisms by which psychoactive drugs increase fall risk (i.e. the influence on gait) are not known. As a high proportion of nursing home residents with dementia are treated with psychoactive drugs, better knowledge of the influence of these medications on fall risk might be useful to prevent further falls. If we know the influence of psychoactive drugs on gait, we can use gait measurements to evaluate the influence of drugs on gait and on subsequent fall risk. We therefore undertook a systematic review of the literature to investigate which psychoactive drugs increase fall risk and what is known about the influence of these drugs on gait in nursing home residents with dementia.

\section{Methods}

\section{Search strategy}

Between 1980 and 31 October 2007 inclusive we performed a broad literature search of Medline, Cinahl, Cochrane, and Psychlit. The following search terms were used: dementia, cognitive impairment, nursing home resident, elderly, older adult; fall, gait, mobility test; drugs, psychoactive medication, psychotropics, antidepressants, benzodiazepines, antipsychotics, sedatives. Randomized controlled trials on drug withdrawal as an intervention and prospective cohort studies published until November 2007 were eligible for inclusion in the review.

\section{Study selection}

Two reviewers independently performed the study selection (CS and TC). Differences of opinion were resolved by discussion between the two reviewers. First, titles and abstracts of identified published articles were reviewed in order to 
determine their relevance. Next, full papers were screened for eligibility. Studies were selected if they met the following criteria: (1) residents with dementia were included in the study population of nursing home residents; and (2) psychoactive medication use was studied. The outcome measures selected were: (1) falls (our primary outcome measure), and (2) gait parameters (our secondary outcome measure, as a possible predictor of risk of falling). If residents with advanced dementia were excluded from participation in a study, we excluded that study from our analysis.

The two reviewers (CS and TC) independently appraised each full text article that passed the first eligibility screening, using a structured form to record our selection criteria. Excluded studies and reasons for exclusion were recorded. The references of all identified relevant studies were individually searched for additional potentially relevant publications. For feasibility reasons, the publication had to be written in English, French, German or Dutch.

\section{Quality assessment}

The two reviewers (CS and TC) assessed the methodological quality of the studies independently, using the nine-item checklist for quality assessment of prospective cohort studies from the Dutch Cochrane Center website. Each item was scored as positive, negative (potential bias), or "not enough information provided," if the paper provided insufficient information on a specific item. Differences in scores were resolved by discussion between the two reviewers, and a third reviewer (AV) was consulted if disagreements could not be resolved.

At item nine on the checklist it was decided if the results of the study were valid and applicable. Item nine was scored as positive if six or more items scored positive. The study was then considered as high quality. Item nine was scored as dubious or negative if fewer than six items scored positive, and the study was then considered as low quality.

\section{Data extraction}

One reviewer (CS) extracted data concerning population characteristics (mean age, gender, cognitive status, dementia severity) and sample size using a structured data collection form. Two reviewers (CS and AV) extracted information and data regarding primary (falls) and secondary (gait parameters) outcome measures, determinants (psychoactive drug use), follow-up period, associations, and adjustments for confounding if reported by the authors, using a standardized form for data extraction from prospective cohort studies from the Dutch Cochrane Center website. In case of disagreement, consensus was achieved by discussion between the two reviewers.

\section{Analysis}

The inter-observer agreement of quality assessment was derived by kappa statistics because of dichotomous values. An inter-observer agreement of $\kappa=0.60-0.80$ represents a good agreement. An inter-observer agreement of $\kappa=0.80-1.00$ represents a very good agreement (Landis and Koch, 1977). 
Table 1. Levels of evidence for prognostic factors

LEVEL OF
EVIDENCE

Strong

Moderate

Limited

Inconclusive
Consistent findings $(\geq 80 \%)$ in at least two high quality cohorts

One high quality cohort and consistent findings $(\geq 80 \%)$ in one or more low quality cohorts

Findings in one cohort or consistent findings in one or more low quality cohorts

Inconsistent findings irrespective of study quality

Pooled risk estimates were not calculated because there was no homogeneity across studies concerning similar drug classes and outcome measures.

Four levels of evidence were defined to assess the strength of evidence for prognostic factors, i.e. strong, moderate, limited and inconclusive (Table 1). Strong evidence was defined as consistent findings $(\geq 80 \%)$ in at least two high quality cohorts (Ariens et al., 2000; Sackett et al., 2000). In the case of dichotomous outcomes, positive clinical relevant findings were considered relative risks (RRs), odds ratios (ORs) or hazard ratios (HRRs) $>2.0$ or $<0.5$ or else significant associations $(\mathrm{p}<0.05)$ (van der Windt et al., 2000). If provided by the authors, positive findings were derived from the multivariate results. If only univariate results were available, we used these findings to determine the level of evidence.

\section{Results}

\section{Search strategy}

The search of the computerized databases identified a total of 499 citations. Based on title and abstract, 63 papers were selected, and a full copy of each paper was applied for and used for the final decision. Screening of the references of all relevant papers resulted in 20 additional studies, making a total of 83 . Of these, 43 papers were excluded because the design was either a case control study, or a case report; 20 were excluded because the study population did not include nursing home residents with dementia or cognitive impairment; and three because they did not describe psychoactive medication as a determinant for falls. In 25 of the 66 excluded papers, falls were not described as an outcome measure. Randomized controlled trials on drug withdrawal as an intervention were not available.

At the end of this selection process, 17 prospective cohort studies were included in this systematic review (see Figure 1).

\section{Quality assessment}

The two reviewers were in agreement on 135 out of 153 items. The interobserver agreement was $\kappa=0.72$. Disagreement occurred mainly because of 


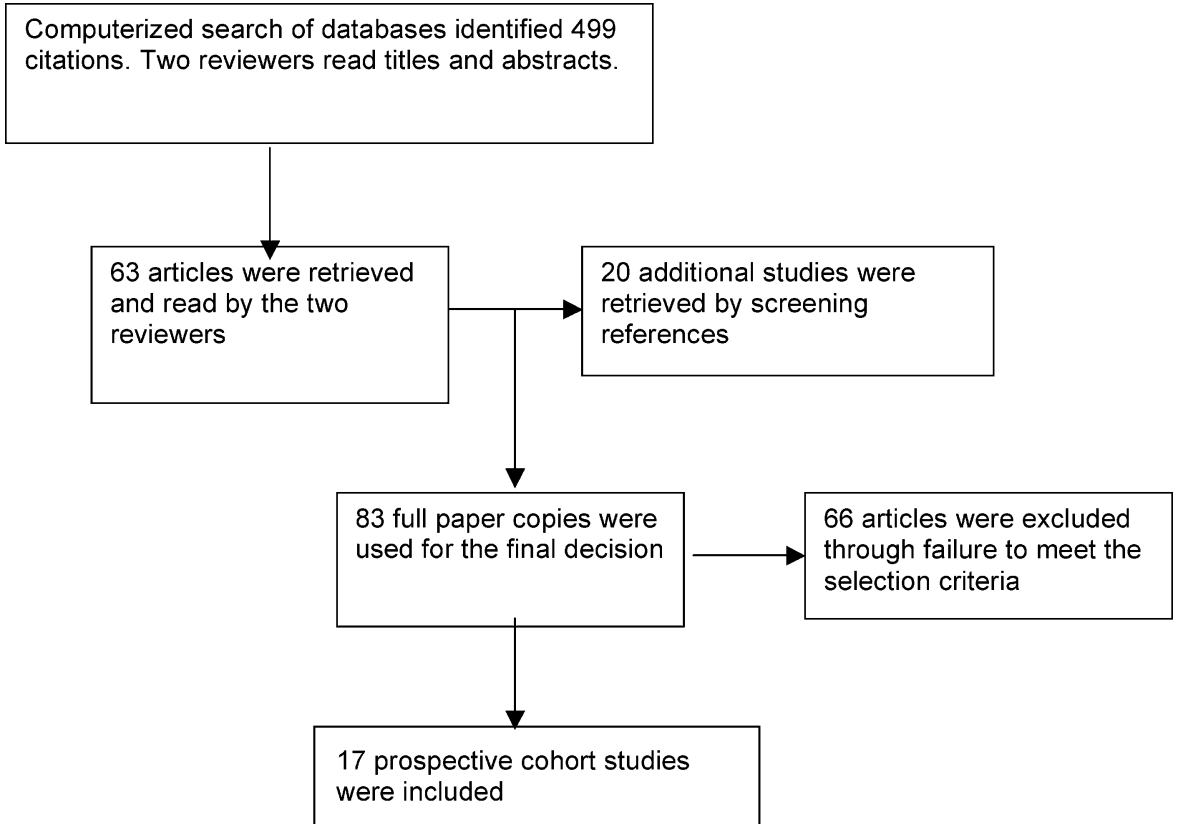

Figure 1. Flow diagram of papers accepted and rejected by the reviewers during the selection procedure

reading errors and interpretation of the methodological criteria list and was readily resolved. The results of the quality assessment are presented in Table 2.

Most methodological shortcomings concerned the following items: an insufficient description of the study population (item 1); an insufficient description of the determinant (item 3); an insufficient description of the outcome (item 4); is the outcome blinded for the determinant? (item 5); an insufficiently long follow-up (item 6); and no information on completers versus loss to follow-up (item 7). Sixteen studies were considered as high quality; one study was considered as low quality.

\section{Study characteristics}

The studies that qualified for inclusion in our review presented their data for total groups of nursing home residents, without a specific sub-group analysis for those with dementia or some cognitive impairment. We therefore analyzed the total groups as this was the nearest possible solution to our initial approach. Table 3 presents a summary of the study characteristics including sample size and population characteristics; determinants of our interest; outcome; crude and adjusted estimates with their 95\% confidence intervals. Table 3 also provides information on adjustments for confounding of the final statistical analysis if reported by the authors.

The sample size varied between $\mathrm{n}=78$ (Rosendahl et al., 2003) and $\mathrm{n}=43,163$ (Avidan et al., 2005). The shortest follow-up period was one month 
Table 2. Results of the quality assessment, showing numeration of the quality items from the Dutch Cochrane Center checklist

\begin{tabular}{|c|c|c|c|c|c|c|c|c|c|c|c|}
\hline \multirow[b]{2}{*}{ COHORT NAME } & \multicolumn{11}{|c|}{ METHODOLOGICAL ITEMS } \\
\hline & 1 & 2 & 3 & 4 & 5 & 6 & 7 & 8 & 9 & QUALIT Y & SCORE \\
\hline Arfken et al., 2001 & 1 & 1 & 1 & 1 & 0 & 1 & ? & 1 & 1 & 7 & \\
\hline Avidan et al., 2005 & 1 & 1 & 1 & 0 & $?$ & 1 & 1 & 1 & 1 & 7 & \\
\hline Capezuti et al., 1996 & 1 & 1 & 1 & 1 & 0 & 1 & 1 & 1 & 1 & 8 & \\
\hline Cooper et al., 2007 & 0 & 1 & 1 & 1 & 0 & 1 & ? & 1 & 1 & 6 & \\
\hline Hien et al., 2005 & 1 & 1 & 1 & 1 & 0 & 0 & 1 & 1 & 1 & 7 & \\
\hline Kiely et al., 1998 & 0 & 1 & 1 & 0 & $?$ & 1 & 1 & 1 & 1 & 6 & \\
\hline Kuchynka et al., 2004 & 0 & 1 & $?$ & 1 & 0 & 1 & ? & 1 & $?$ & 4 & \\
\hline Lipsitz et al., 1991 & 1 & 1 & 1 & 1 & 0 & 1 & 1 & 1 & 1 & 8 & \\
\hline Lord et al., 2003 & 0 & 1 & 0 & 1 & 0 & 1 & 1 & 1 & 1 & 6 & \\
\hline Ray et al., 2000 & 1 & 1 & 1 & 1 & 0 & 1 & 1 & 1 & 1 & 8 & \\
\hline Ray et al., 2002 & 1 & 1 & 1 & 1 & 0 & 1 & 1 & 1 & 1 & 8 & \\
\hline Rosendahl et al., 2002 & 1 & 1 & 1 & 1 & 0 & 1 & 0 & 1 & 1 & 7 & \\
\hline Ruthazer and Lipzitz, 1993 & 1 & 1 & 1 & 1 & 0 & 0 & 1 & 1 & 1 & 7 & \\
\hline Thapa et al., 1995 & 1 & 1 & 1 & 1 & 0 & 1 & 1 & 1 & 1 & 8 & \\
\hline Thapa et al., 1996 & 1 & 1 & 1 & 1 & $?$ & 1 & 1 & 1 & 1 & 8 & \\
\hline Thapa et al., 1998 & 1 & 1 & 1 & 1 & 0 & 1 & 1 & 1 & 1 & 8 & \\
\hline van Doorn et al., 2003 & 1 & 1 & 1 & 1 & 0 & 1 & ? & 1 & 1 & 7 & \\
\hline
\end{tabular}

Quality items: sufficient description study population (item 1); exclusion of selection bias (item 2); sufficient description determinant (item 3); sufficient description outcome (item 4); is the outcome blinded for the determinant? (item 5); sufficiently long follow-up (item 6); information on completers versus loss to follow-up (item 7); information on confounders (item 8); validity results (item 9). Items are scored as positive scores (1), negative (0), or unclear/insufficient information (?).

(Ruthazer and Lipsitz, 1993; Hien et al., 2005), and the longest was two years (Lipsitz et al., 1991; van Doorn et al., 2003).

\section{FALLS}

Most studies ascertained falls from medical records or nursing home charts and from incidence reports (Ruthazer and Lipsitz, 1993; Thapa et al., 1995; 1996; 1998; Ray et al., 2000; 2002; Arfken et al., 2001; Lord et al., 2003; Hien et al., 2005). In one study falls were ascertained from a subject interview (Lipsitz et al., 1991), and in another from the registration form and reported to a study nurse (Rosendahl et al., 2003). In four studies falls were ascertained only from incidence reports (Capezuti et al., 1996; Kuchynka et al., 2004) or nursing home charts (Kiely et al., 1998; van Doorn et al., 2003; Avidan et al., 2005; Cooper et al., 2007).

\section{GAIT PARAMETERS}

None of the studies described gait parameters as outcome measure for psychoactive drug use. Some studies described gait parameters as determinants 
Table 3. Summary of study characteristics

\begin{tabular}{|c|c|c|c|c|c|c|}
\hline $\mathrm{COHORT}$ & P O P U LAT ION & DETERMINANTS & $\begin{array}{l}\text { OUTCOME } \\
\text { MEASURES }\end{array}$ & $\begin{array}{l}\text { C RUDE } \\
\text { ESTIMATES } \\
\text { A ND } 95 \% \text { C I }\end{array}$ & $\begin{array}{l}\text { A D J US TED } \\
\text { EST IMATES } \\
\text { AND } 95 \% \text { C I }\end{array}$ & NOTES \\
\hline $\begin{array}{l}\text { Arfken } \text { et al. } \\
\quad 2001 \\
Q=7\end{array}$ & $\begin{array}{l}\mathrm{N}=368 \\
\text { Memory problems } \\
\quad 43.7 \% \\
\text { Age } \pm 80 \\
\text { Female } \pm 70 \%\end{array}$ & $\begin{array}{l}\text { Antidepressant (Selective } \\
\text { serotonine-reuptake } \\
\text { inhibitor and Non- } \\
\text { Selective } \\
\text { serotonine-reuptake } \\
\text { inhibitor) use }\end{array}$ & $\begin{array}{l}\text { Falls (incident } \\
\text { reports and fall } \\
\text { logs) }\end{array}$ & & $\begin{array}{l}\text { Selective } \\
\quad \text { serotonine-reuptake } \\
\text { inhibitor } \\
\text { OR }=2.01 \text { (1.23-3.28) } \\
\text { Non- Selective } \\
\quad \text { serotonine-reuptake } \\
\text { inhibitor } \\
\text { OR }=1.40 \text { (0.65-3.03) } \\
\text { Selective serotonine- } \\
\text { reuptake inhibitor } \\
\text { OR }=1.77(1.0-3.13)\end{array}$ & $\begin{array}{l}\text { Adjusted for age, number of medications, } \\
\text { number of diagnoses, gender, memory } \\
\text { problems, restraints }\end{array}$ \\
\hline $\begin{array}{l}\text { Avidan et al., } \\
\quad 2005 \\
Q=7\end{array}$ & $\begin{array}{l}\mathrm{N}=34163 \\
\text { Moderately-very } \\
\quad \text { severely cognitive } \\
\quad \text { impaired } 77.3 \% \\
\text { Age } 84.2(7.7) \\
\text { Female } 76.5 \%\end{array}$ & Hypnotic use & $\begin{array}{l}\text { Falls (The } \\
\text { Resident } \\
\text { Assessment } \\
\text { Instrument/ } \\
\text { Minimum Data } \\
\text { Set) }\end{array}$ & $\mathrm{OR}=1.29(1.13-1.48)$ & $\mathrm{OR}=1.13(0.98-1.30)$ & $\begin{array}{l}\text { Adjusted for age, sex, functional status, } \\
\text { cognitive status, intensity of resource } \\
\text { utilization, burden of illness, number } \\
\text { of medications taken, emergency } \\
\text { department visits, and new admission }\end{array}$ \\
\hline $\begin{array}{l}\text { Capezuti et al., } \\
\quad \begin{array}{l}1996 \\
\mathrm{Q}=8\end{array}\end{array}$ & $\begin{array}{l}\mathrm{N}=322 \\
\text { Severe cognitive } \\
\quad \text { impaired } 27.6 \% \\
\text { Age } \pm 84(7.3)\end{array}$ & Psychoactive drug use & $\begin{array}{l}\text { Falls (incidence } \\
\text { reports) }\end{array}$ & $\mathrm{OR}=1.78(1.14-2.79)$ & Not provided & $\begin{array}{l}\text { Table provides unadjusted estimates, the } \\
\text { text shows the same figures as adjusted } \\
\text { estimates }\end{array}$ \\
\hline $\begin{array}{l}\text { Cooper et al., } \\
\quad \begin{array}{l}2007 \\
Q=6\end{array}\end{array}$ & $\begin{array}{l}\mathrm{N}=177 \\
\text { Age } 81.8(10.7) \\
\text { Female } 79 \%\end{array}$ & No. Psychotropic drug use & $\begin{array}{l}\text { Falls (patient } \\
\text { charts) }\end{array}$ & $\begin{array}{l}1 \text { psychotropic } \\
\mathrm{RR}=1.8(1.21-2.84) \\
2 \text { psychotropics } \\
\mathrm{RR}=3.2(2.25-4.51) \\
3 \text { psychotropics } \\
\mathrm{RR}=6.7(4.15-8.53) \\
4 \text { psychotropics } \\
\mathrm{RR}=10.3(6.91-12.8)\end{array}$ & & \\
\hline $\begin{array}{l}\text { van Doorn et al., } \\
\quad 2003 \\
Q=7\end{array}$ & $\begin{array}{l}\mathrm{N}=2015 \\
\text { Demented } 48.2 \% \\
\text { Age } 81.4(7.6) \\
\text { Female } 70.4 \%\end{array}$ & $\begin{array}{l}\text { Antipsychotic, Antianxiety, } \\
\text { Antidepressant } \\
\text { medication use }\end{array}$ & $\begin{array}{l}\text { Falls (nursing } \\
\text { home charts) }\end{array}$ & $\begin{array}{l}\text { Antipsychotics } \\
\mathrm{RR}=1.83(1.48-2.26) \\
\text { Antianxiety medication } \\
\mathrm{RR}=1.32(1.01-1.72) \\
\text { Antidepressants } \\
\mathrm{RR}=1.44(1.08-1.90)\end{array}$ & Not provided & \\
\hline
\end{tabular}




\section{Hien et al., $2005 \mathrm{~N}=898$ \\ $\mathrm{Q}=7 \quad$ Mean age 85.7 \\ Female $76 \%$}

Kiely et al., $1998 \mathrm{~N}=18855$

$\mathrm{Q}=6$

$$
\begin{aligned}
& \text { Cognitive impaired } \\
& 82 \% \\
& \text { Median age } 87 \\
& \text { Female } 84 \%
\end{aligned}
$$

\section{Kuchynka et al., $\mathrm{N}=314$}

$2004 \quad$ Demented 31.8\%

$\mathrm{Q}=4 \quad$ Age \pm 82

$\begin{array}{ll}\text { Lipsitz et al., } & \mathrm{N}=126\end{array}$

1991 Cognitive impaired

$\mathrm{Q}=8 \quad \mathrm{n}=40$

Mean age 87

Female $61 \%$
Antidepressant, Sedatives/ anxiolytics, Typical

antipsychotic,

Olanzapine, Risperidone

use

Antipsychotic

Antianxiety medication use Resident

Assessment

Instrument/

Minimum Dat

Set)

Benzodiazepine use

\section{Falls (in}

reports)

Antidepressant and

Sedative medication use

\section{Falls}

Falls (incidence Antidepressant

and computer

$\mathrm{OR}=5.67(1.57-20.48$

Antidepressants

Antidepressants

$\mathrm{HR}=1.56(1.19-2.04) \quad \mathrm{HR}=1.45$ (1.09-1.93)

Sedatives/anxiolytics

$\mathrm{HR}=1.37$

$\mathrm{HR}=1.19(0.94-1.50)$

Typical antipsychotic Typical antipsychotic

$\mathrm{HR}=1.48(0.96-2.26) \quad \mathrm{HR}=1.35(0.87-2.09)$

Olanzapine

Olanzapine

$\mathrm{HR}=2.35(1.43-3.87) \quad \mathrm{HR}=1.74(1.04-2.90)$

Risperidone

$\mathrm{HR}=1.74$
Risperidone

(0.75-3.87) $\quad \mathrm{HR}=1.32(0.57-3.06)$

Antipsychotic

Not provided

$\mathrm{OR}=1.21(1.11-1.33)$

Antianxiety

reports, medical Sedatives

records, and $\mathrm{OR}=1.95(0.89-4.30)$

subject

interview)
Adjusted for other psychotropics in the model, age, sex, type of residential care facility, length of stay, residential

Classification Scale score, Implicit illness severity scale, MMSE-score, Parkinson's disease, previous falls, static balance score
Prevalence: $27 \%$ of the fallers were benzodiazepine users, $25 \%$ of the non-fallers were benzodiazepine users

Adjusted for Medication variables: cardiovascular, neuroleptic, sedative, non-steroidal anti-inflammatory;

Physical examination variables: visua acuity, impaired hearing, impaired vibration sensation, impaired position sensation, impaired touch sensation, lower extremity muscle weakness, increased muscle tone, apraxia combing hair, dysmetria, orthopedic deformity, orthostatic dizziness, orthostatic hypotension; Functional examination variables: unsteady (eyes open/closed), unsteady (sternal push), intermittent turning, unsteady turning, chair stand, broad stance, hesitant gait initiation, reduced step height, reduced step length, step asymmetry, step

discontinuity, path deviation, trunkal instability; Continuous functional gait variables: chair stand, $>25$ steps $/ 20$ foot walk, $>18.8 \mathrm{sec} / 20$ foot walk, $>9.1 \mathrm{sec}$ to turn $360,>12$ steps to turn 
Table 3. Continued

\begin{tabular}{|c|c|c|c|c|c|c|}
\hline $\mathrm{COHORT}$ & POPULATION & DETERMINANTS & $\begin{array}{l}\text { OUTCOME } \\
\text { MEASURES }\end{array}$ & $\begin{array}{l}\text { CRUDE } \\
\text { ESTIMATES } \\
\text { AND } 95 \% \text { C I }\end{array}$ & $\begin{array}{l}\text { A D J U S T E D } \\
\text { ES T IM ATES } \\
\text { AND } 95 \% \text { C I }\end{array}$ & NOTES \\
\hline $\begin{array}{l}\text { Lord et al., } 2003 \\
\mathrm{Q}=6\end{array}$ & $\begin{array}{l}\mathrm{N}=228 \\
\mathrm{~N}=\text { demented? } \\
\text { Age } 85(7.4) \\
\text { Females } 72 \%\end{array}$ & $\begin{array}{l}\text { Sedatives } \\
\text { Antipsychotics } \\
\text { Antidepressants } \\
\text { Any psychotropic } \geq 2 \\
\quad \text { psychotropics }\end{array}$ & $\begin{array}{l}\text { Falls (incidence } \\
\text { reports and } \\
\text { medical records) }\end{array}$ & $\begin{array}{l}\text { Sedatives } \\
\text { IRR }=1.27(1.01-1.60) \\
\text { Antipsychotics } \\
\text { IRR }=1.27(0.92-1.75) \\
\text { Antidepressants } \\
\text { IRR }=1.34(1.05-1.72) \\
\text { Any psychotropic } \\
\text { IRR }=1.47(1.20-1.81) \\
\quad \geq 2 \text { psychotropics } \\
\text { IRR }=1.30(1.00-1.69)\end{array}$ & $\begin{array}{l}\text { Any psychotropic } \\
\mathrm{IRR}=1.36(1.05-1.76)\end{array}$ & $\begin{array}{l}\text { Adjusted for age, sex, resident } \\
\text { classification score, Implicit illness } \\
\text { severity score, SMMSE, Parkinson's } \\
\text { disease, stroke, day incontinence, night } \\
\text { incontinence, osteoarthritis in } \\
\text { either/both knees, fall in previous year, } \\
\text { walking aid, } \geq 4 \text { medications, visual } \\
\text { contrast sensitivity, proprioception, } \\
\text { quadriceps strength, reaction time, } \\
\text { sway-on floor, sway-on foam, static } \\
\text { balance, sit-to-stand ability }\end{array}$ \\
\hline $\begin{array}{l}\text { Thapa et al., } \\
\quad 1998 \\
\text { Ray et al., } 2000 \\
\text { Ray et al., } 2002 \\
\text { Q=8 }\end{array}$ & $\begin{array}{l}\mathrm{N}=2428(\text { Ray } 2000 \\
\mathrm{n}=2510) \\
\text { Mean age } 82 \\
\text { Major cognitive } \\
\text { impairment } 22 \% \\
\text { Female } 75 \%\end{array}$ & $\begin{array}{l}\text { Benzodiazepine } \\
\text { Antidepressant (Tricyclic } \\
\text { antidepressants, Selective } \\
\text { serotonine-reuptake } \\
\text { inhibitor, and Trazodone } \\
\text { use) } \\
\text { Antipsychotic and other } \\
\text { sedatives/hypnotic } \\
\text { specific drug use }\end{array}$ & $\begin{array}{l}\text { Falls (incidence } \\
\text { reports and } \\
\text { medical records) }\end{array}$ & $\begin{array}{l}\text { Tricyclic } \\
\quad \text { antidepressant } \\
\mathrm{RR}=2.4(2.1-2.6) \\
\text { Nortriptyline } \\
\mathrm{RR}=2.3(2.0-2.5) \\
\text { Amitriptyline } \\
\mathrm{RR}=2.2(2.0-2.5) \\
\text { Doxepin } \\
\mathrm{RR}=2.4(2.1-2.8) \\
\mathrm{Imipramine} \\
\mathrm{RR}=2.6(2.2-3.1) \\
\text { Other } \\
\mathrm{RR}=3.1(2.5-3.9) \\
\end{array}$ & $\begin{array}{l}\text { Tricyclic antidepressant } \\
\mathrm{RR}=2.0(1.8-2.2) \\
\text { Nortriptyline } \\
\mathrm{RR}=2.0(1.8-2.3) \\
\text { Amitriptyline } \\
\mathrm{RR}=1.9(1.7-2.1) \\
\text { Doxepin } \\
\mathrm{RR}=2.0(1.7-2.3) \\
\mathrm{Imipramine} \\
\mathrm{RR}=2.2(1.8-2.6) \\
\text { Other } \\
\mathrm{RR}=2.4(1.9-3.0) \\
\mathrm{Selective} \mathrm{serotonine-} \\
\text { reuptake inhibitors } \\
<20 \mathrm{mg} \\
\mathrm{RR}=1.5(1.3-1.7) \\
\geq 20 \mathrm{mg} \\
\mathrm{RR}=1.9(1.7-2.2) \\
\mathrm{Selective} \mathrm{serotonine-} \\
\text { reuptake inhibitors } \\
\mathrm{RR}=1.8(1.6-2.0) \\
\text { Paroxetine } \\
\mathrm{RR}=1.7(1.5-1.9) \\
\text { Fluoxetine } \\
\mathrm{RR}=1.8(1.6-2.1) \\
\mathrm{Sertraline} \\
\mathrm{RR}=1.8(1.5-2.1) \\
\text { Trazodone }<50 \mathrm{mg} \\
\mathrm{RR}=1.5(1.2-1.8) \\
\geq 50 \mathrm{mg} \\
\mathrm{RR}=1.1(1.0-1.3)\end{array}$ & $\begin{array}{l}\text { Adjusted for age, gender, race, time since } \\
\text { admission to facility and since cohort } \\
\text { entry, body mass index, ambulatory } \\
\text { status, number of activities of daily } \\
\text { living with total dependency, } \\
\text { incontinence, cognitive impairment, } \\
\text { physical restraint use, past falls, use of } \\
\text { anticonvulsants, antiparkinson drugs, } \\
\text { antidepressants, antipsychotics, and } \\
\text { other sedatives }\end{array}$ \\
\hline
\end{tabular}


benzodiazepines

$\mathrm{RR}=1.02(0.95-1.10)$

Tricyclic antidepressan

$$
\leq 10 \mathrm{mg}
$$

$\mathrm{RR}=1.2(1.0-1.5)$

$11-25 \mathrm{mg}$

$\mathrm{RR}=2.0(1.8-2.3)$

26-50 mg

$\mathrm{RR}=2.1(1.8-2.3)$

$>50 \mathrm{mg}$

$\mathrm{RR}=2.4(2.1-2.8)$

Current

benzodiazepines

$\mathrm{RR}=1.44(1.33-1.56)$

Dose current users

$\leq 2 \mathrm{mg}$

$\mathrm{RR}=1.30(1.12-1.52)$

2.01-4 mg

$\mathrm{RR}=1.34(1.20-1.51)$

$4.01-8 \mathrm{mg}$

$\mathrm{RR}=1.38(1.20-1.51)$

$\mathrm{RR}=2.21(1.89-2.60)$

Days since start of

use $<7$

$\mathrm{RR}=2.96(2.33-3.75)$

7-29

$\mathrm{RR}=2.23(1.64-3.03)$ $\geq 30$

$\mathrm{RR}=1.30(1.17-1.44)$

Elimination half-life,

hours $<12$

$\mathrm{RR}=1.15(0.94-1.40)$

$$
\text { 12-23 }
$$

$\mathrm{RR}=1.44(1.33-1.59)$

$$
\geq 24
$$

$\mathrm{RR}=1.73(1.40-2.14)$

Current benzodiazepine

use

$\mathrm{RR}=1.38(1.25-1.51)$

Elimination half-life,

$$
\text { hours }<12
$$

$\mathrm{RR}=0.90(0.70-1.17)$

$$
\text { 12-23 }
$$


Table 3. Continued

\begin{tabular}{|c|c|c|c|c|c|c|}
\hline $\mathrm{COHORT}$ & POPULATION & DETERMINANTS & $\begin{array}{l}\text { OUTCOME } \\
\text { MEASURES }\end{array}$ & $\begin{array}{l}\text { C R U D E } \\
\text { ESTIMATES } \\
\text { AND } 95 \% \text { C I }\end{array}$ & $\begin{array}{l}\text { A D J USTED } \\
\text { ESTIMATES AND } \\
95 \% \text { C I }\end{array}$ & NOTES \\
\hline $\begin{array}{l}\text { Rosendahl et al., } \\
\quad 2003 \\
Q=7\end{array}$ & $\begin{array}{l}\mathrm{N}=78 \\
\text { Demented } 47 \% \\
\text { Age } 81(6) \\
\text { Female } 72 \%\end{array}$ & $\begin{array}{l}\text { Tranquilizers/sedatives } \\
\text { Antidepressant use }\end{array}$ & $\begin{array}{l}\text { Falls (register form } \\
\text { and reported to } \\
\text { study nurse) }\end{array}$ & $\begin{array}{l}\text { Tranquillizers/ } \\
\quad \text { sedatives } \\
\mathrm{HR}=1.66(0.93-2.96) \\
\text { Antidepressants } \\
\mathrm{HR}=1.93(1.05-3.52)\end{array}$ & $\begin{array}{l}\mathrm{RR}=1.43(1.29-1.59) \geq 24 \\
\text { Current benzodiazepine use } \\
\mathrm{RR}=1.83(1.55-2.15) \\
\text { Elimination half-life, hours }<12 \\
\mathrm{RR}=2.19(1.59-3.03) 12-23 \\
\mathrm{RR}=1.68(1.39-2.02) \geq 24 \\
\mathrm{RR}=1.80(1.14-2.83) \\
\text { Not provided }\end{array}$ & \\
\hline $\begin{array}{l}\text { Ruthazer and } \\
\quad \text { Lipsitz, } 1993 \\
Q=7\end{array}$ & $\begin{array}{l}\mathrm{N}=635 \\
\mathrm{~N}=\text { demented ? } \\
\text { Mean age } 88.7 \\
\text { Female } 77 \%\end{array}$ & $\begin{array}{l}\text { Antidepressant, } \\
\text { Antipsychotic, } \\
\text { Benzodiazepine use }\end{array}$ & $\begin{array}{l}\text { Falls } \\
\text { (computerized } \\
\text { documentation } \\
\text { systems and } \\
\text { chart reviews) }\end{array}$ & $\begin{array}{l}\text { Antidepressants } \\
\quad \text { (women) } \\
\mathrm{OR}=1.95(1.02-3.70)\end{array}$ & $\begin{array}{l}\text { Adjusted estimate } \\
\text { Antidepressants (women) } \\
\mathrm{OR}=1.84(0.91-3.69)\end{array}$ & $\begin{array}{l}\text { Stratified for sex. Adjusted } \\
\text { for age and fall history }\end{array}$ \\
\hline $\begin{array}{l}\text { Thapa et al., } \\
1995 \\
Q=8\end{array}$ & $\begin{array}{l}\mathrm{N}=282 \\
\text { Moderate - severely } \\
\quad \text { cognitive impaired } \\
\quad 68.8 \% \\
\text { Age } 80.9 \\
\text { Female } 72 \%\end{array}$ & $\begin{array}{l}\text { Any psychotropic drug } \\
\text { Antipsychotics } \\
\text { Benzodiazepines } \\
\text { Cyclic antidepressants } \\
\text { Other anxiolytics/hypnotics } \\
\text { Multiple psychotropic } \\
\quad \text { drug use }\end{array}$ & $\begin{array}{l}\text { Recurrent falls } \geq 2 \\
\quad \text { (incidence } \\
\text { reports and } \\
\text { nursing home } \\
\text { charts) }\end{array}$ & $\begin{array}{l}\text { Any psychotropic drug } \\
\text { IDR }=1.67(1.10-2.5) \\
\text { Antipsychotics } \\
\text { IDR }=1.54(0.88-2.7) \\
\text { Benzodiazepines } \\
\text { IDR }=1.70(0.96-2.9) \\
\text { Cyclic antidepressants } \\
\text { IDR }=1.98(0.97-4.0) \\
\text { Other anxiolytics } / \\
\text { hypnotics } \\
\text { IDR }=1.26(0.57-2.7) \\
\text { Multiple psychotropic } \\
\text { drugs } \\
\text { IDR }=1.89(1.10-3.2)\end{array}$ & $\begin{array}{l}\text { Adjusted incidence density } \\
\quad \text { ratios } \\
\text { Any psychotropic drug } \\
\text { IDR }=1.97(1.28-3.05) \\
\text { Antipsychotics } \\
\text { IDR }=1.48(0.79-2.78) \\
\text { Benzodiazepines } \\
\text { IDR }=2.10(1.17-3.76) \\
\text { Cyclic antidepressants } \\
\text { IDR }=2.92(1.39-6.16) \\
\text { Other anxiolytics / hypnotics } \\
\text { IDR }=1.23 \text { (0.55-2.76) } \\
\text { Multiple psychotropic drugs } \\
\text { IDR }=2.57(1.45-4.57)\end{array}$ & $\begin{array}{l}\text { Adjusted for age, assisted } \\
\text { activities of daily living, } \\
\text { balance score, symptoms of } \\
\text { dementia and depression, other } \\
\text { psychotropic drug use. }\end{array}$ \\
\hline $\begin{array}{l}\text { Thapa et al., } \\
\begin{array}{l}1996 \\
Q=8\end{array}\end{array}$ & $\begin{array}{l}\mathrm{N}=503 \\
\text { Moderate and Severe } \\
\text { cognitive impaired } \\
\mathrm{n}=218 \\
\text { Age } 37.2 \% \geq 85 \\
\text { Female } 73 \%\end{array}$ & $\begin{array}{l}\text { Psychotropic drug use } \\
\text { (Antipsychotics, } \\
\text { Benzodiazepines, Cyclic } \\
\text { antidepressants/ } \\
\text { Trazodone, other } \\
\text { Hypnotics/ anxiolytics) }\end{array}$ & $\begin{array}{l}\text { Injurious falls } \\
\text { (incidents } \\
\text { reports and } \\
\text { nursing home } \\
\text { charts) }\end{array}$ & $\begin{array}{l}\text { Unadjusted incidence } \\
\text { rates, per } 100 \text { person } \\
\text { years } \\
\text { Psychotropic drugs } \\
\text { IDR }=23.4\end{array}$ & $\begin{array}{l}\text { Adjusted incidence density } \\
\quad \text { ratios } \\
\text { Psychotropic drugs } \\
\text { IDR }=2.49(1.43-4.33)\end{array}$ & $\begin{array}{l}\text { Adjusted for age, gender, BMI, } \\
\text { cognitive impairment }\end{array}$ \\
\hline
\end{tabular}

Note: $\mathrm{Q}=$ quality score; $\mathrm{OR}=$ odds ratio; $\mathrm{RR}=$ relative risk; $\mathrm{HR}=$ hazard ratio; $\mathrm{IRR}=$ incidence rate ratio; $\mathrm{IDR}=$ incidence density ratio. 
for falls (Lipsitz et al., 1991; Lord et al., 2003; Rosendahl et al., 2003; van Doorn et al., 2003; Kuchynka et al., 2004).

\section{PSYCHOACTIVE DRUG USE}

In most studies drug use is the determinant of primary interest (Ruthazer and Lipsitz, 1993; Thapa et al., 1995; 1996; 1998; Ray et al., 2000; 2002; Arfken et al., 2001; Avidan et al., 2005; Hien et al., 2005; Cooper et al., 2007). In other studies psychoactive drugs are studied among other risk factors to develop or to evaluate a fall risk model (Lipsitz et al 1991; Kiely et al., 1998; Lord et al., 2003; Rosendahl et al., 2003). One study described the effect of restraint use on falls, with drug use being a confounder in their multiple logistic regression model (Capezuti et al., 1996). In another study, dementia is the factor of primary interest. Other variables, including antipsychotic, anti-anxiety and antidepressant drug use were evaluated as potential confounders (van Doorn et al., 2003).

The Minimum Data Set (MDS; Morris et al., 1990) was used by most studies to ascertain psychoactive drug use (Kiely et al., 1998; van Doorn et al., 2003; Avidan et al., 2005). Other studies used pharmacy records (Arfken et al., 2001; Cooper et al., 2007), medical records (Lord et al., 2003; Hien et al., 2005), or medication administration records (Lipsitz et al 1991; Ruthazer and Lipsitz, 1993; Thapa et al., 1995; 1996; Capezuti et al., 1996; Ray et al., 2000; 2002). Some studies provided information on dose or duration of use (Capezuti et al., 1996; Thapa et al., 1998; Ray et al., 2000; 2002). In one study, psychoactive drug use was calculated as the proportion of days when psychoactive drugs were used divided by the number of days the resident was present in the nursing home; drug use was categorized by degrees of use as "none," "some" (1-98 days), and "all" (daily use) (Capezuti et al., 1996). In two studies, benzodiazepine use was classified for each day of follow-up as "current" (taken that day), "recent", or "none" (Ray et al., 2000; 2002). One study considered dose, duration and elimination half-life in relation to falls. Elimination half-life was also considered in relation to daytime and night-time falls (Ray et al., 2000). In one study any recent change in medication and the time when medications were taken in relation to the fall were recorded, and a blood sample was obtained to check any relevant drug level (Lipsitz et al., 1991). In only one study is it unclear as to how drug use was ascertained (Kuchynka et al., 2004).

\section{Associations}

Eleven publications presented the associations between psychoactive drug use and falls in adjusted estimates: OR (Lipsitz et al., 1991; Ruthazer and Lipsitz, 1993; Arfken et al., 2001; Avidan et al., 2005); RR (Thapa et al., 1998; Ray et al., 2000; 2002); incidence density ratio (IDR; Thapa et al., 1995; 1996), HR (Hien et al., 2005), and incidence rate ratio (IRR; Lord et al., 2003). One study stratified for sex (Ruthazer and Lipsitz, 1993). Five publications presented only crude estimates of the associations between psychoactive drug use and falls: OR (Capezuti et al., 1996), RR (Kiely et al., 1998; van Doorn et al., 2003; Cooper et al., 2007), and HR (Rosendahl et al., 2003). One publication only presented 
the prevalence of fallers among benzodiazepine users and among non-users (Kuchynka et al., 2004).

All publications presented their results for residents with and without dementia together. None of the studies provided a sub-group analysis of the estimates in the population of nursing home residents with dementia. In five studies, it was unclear which proportion of the population had dementia (Ruthazer and Lipsitz, 1993; Ray et al., 2000; Lord et al., 2003; Hien et al., 2005; Cooper et al., 2007). In two publications, the estimates for the whole cohort - both those in nursing homes and in intermediate care facilities - were given. In these studies there was no sub-group analysis of the estimates of the proportion of the population in the nursing homes (Lord et al., 2003; Hien et al., 2005).

\section{Level of evidence}

The heterogeneity of the study population and determinants necessitated a qualitative summary of the results. Table 4 presents a summary of the available evidence for the use of psychoactive drugs and its association with falls in nursing home populations including residents with dementia. Three papers classified all psychoactive drugs together, regardless of specific drug class (Capezuti et al., 1996; Thapa et al., 1996; Cooper et al., 2007). All other studies presented data by drug class; they are presented both in the psychoactive and in the individual drug summary of the results. The results of studies that presented data on benzodiazepines, hypnotics, sedatives and anti-anxiety drugs were also summarized together and for the individual drug classes. Three papers provided data on the antidepressant class (Thapa et al., 1995; 1998; Arfken et al., 2001); they are presented in both the antidepressant and the individual antidepressant class summary.

\section{ANY PSYCHOACTIVE DRUG}

The overall evidence that the use of any psychoactive drug increases fall risk in nursing home residents with dementia is inconclusive. The reported strength of the associations varied widely (ORs and RRs 0.90-7.6). Positive findings were found in 28 out of $42(67 \%)$ of the studies. The evidence that any psychoactive drug increases recurrent falls is limited. We found only one study in which the use of psychoactive drugs increased the risk of recurrent falls (Thapa et al., 1995). The evidence for injurious falls is strong. Positive findings were found in two studies $(\mathrm{n}=503$, IDR 2.49 and $\mathrm{n}=368$, OR 1.77) (Thapa et al., 1996; Arfken et al., 2001).

\section{BENZODIAZEPINES AND OTHER HYPNOTIC, SEDATIVE OR}

ANTI-ANXIETY DRUGS

For the whole spectrum of benzodiazepines or any other hypnotic, sedative or anti-anxiety drug, we found that the overall evidence that these drugs increase the risk of falls or recurrent falls is inconclusive. Positive findings for the risk of falls were found in five out of 11 cohorts (45\%) (range of ORs and RRs 1.132.4) (Thapa et al., 1995; 1998; Kiely et al., 1998; van Doorn et al., 2003; Lord 
Table 4. Qualitative summary of the available evidence

\begin{tabular}{|c|c|c|c|c|c|c|c|c|c|}
\hline $\begin{array}{l}\text { PSYCHOACTIVE } \\
\text { DRUGS }\end{array}$ & OUTCOME & $\begin{array}{l}\text { COHORT } \\
\text { ASSESSED }\end{array}$ & +FINDINGS & $\begin{array}{l}+ \text { HIGH } \\
\text { QUALITY }\end{array}$ & $\begin{array}{l}+ \text { LOW } \\
\text { QUALITY }\end{array}$ & - FINDINGS & $\begin{array}{l}-\mathrm{HIGH} \\
\text { QUALITY }\end{array}$ & $\begin{array}{l}- \text { LOW } \\
\text { QUALITY }\end{array}$ & $\begin{array}{l}\text { LEVEL OF } \\
\text { EVIDENCE }\end{array}$ \\
\hline \multirow[t]{3}{*}{ Any psychoactive drug } & Falls & 42 & $28 / 42(67 \%)$ & 28 & - & $14 / 42(33 \%)$ & 13 & 1 & Inconclusive \\
\hline & Recurrent falls & 1 & $1 / 1(100 \%)$ & 1 & - & - & - & - & Limited yes \\
\hline & Injurious falls & 2 & $2 / 2(100 \%)$ & 2 & - & - & - & - & Strong yes \\
\hline Hypnotics & Falls & 11 & $5 / 11(45 \%)$ & 5 & & $6 / 11(55 \%)$ & 5 & 1 & Inconclusive \\
\hline Sedatives & Recurrent falls & 2 & $1 / 2(50 \%)$ & - & - & $1 / 2(50 \%)$ & - & - & Inconclusive \\
\hline \multicolumn{10}{|l|}{ Anti-anxiety } \\
\hline \multirow{2}{*}{ Hypnotics } & Falls & 2 & - & - & - & $2 / 2(100 \%)$ & 2 & - & Strong no \\
\hline & Recurrent falls & 1 & - & - & - & $1 / 1(100 \%)$ & 1 & - & Limited no \\
\hline Sedatives & Falls & 4 & $1 / 4(25 \%)$ & 1 & - & $3 / 4(75 \%)$ & 3 & - & Inconclusive \\
\hline Anti-anxiety & Falls & 2 & $2 / 2(100 \%)$ & 2 & - & - & - & - & Strong yes \\
\hline \multirow[t]{4}{*}{ Benzodiazepines } & Falls & 3 & $2 / 3(67 \%)$ & 2 & - & $1 / 3(33 \%)$ & - & 1 & Inconclusive \\
\hline & Recurrent falls & 1 & $1 / 1(100 \%)$ & 1 & - & - & - & - & Limited yes \\
\hline & Daytime falls & 1 & $1 / 1(100 \%)$ & 1 & - & - & - & - & Limited yes \\
\hline & Night-time falls & 1 & $1 / 1(100 \%)$ & 1 & - & - & - & - & Limited yes \\
\hline \multirow{3}{*}{$\begin{array}{l}\text { Benzodiazepine use } \\
\text { elimination } \\
\text { half-life }<12 \text { hours }\end{array}$} & Falls & 1 & - & - & - & $1 / 1(100 \%)$ & 1 & - & Limited no \\
\hline & Daytime falls & 1 & - & - & - & $1 / 1(100 \%)$ & 1 & - & Limited no \\
\hline & Night-time falls & 1 & $1 / 1(100 \%)$ & 1 & - & - & - & - & Limited yes \\
\hline \multirow{3}{*}{$\begin{array}{l}\text { Benzodiazepine use } \\
\text { elimination } \\
\text { half-life } \geq 12 \text { hours }\end{array}$} & Falls & 1 & $1 / 1(100 \%)$ & 1 & - & - & - & - & Limited yes \\
\hline & Daytime falls & 1 & $1 / 1(100 \%)$ & 1 & - & - & - & - & Limited yes \\
\hline & Night-time falls & 1 & $1 / 1(100 \%)$ & 1 & - & - & - & - & Limited yes \\
\hline \multirow[t]{2}{*}{ Antipsychotics } & Falls & 7 & $3 / 7(43 \%)$ & 3 & - & $4 / 7(57 \%)$ & 3 & - & Inconclusive \\
\hline & Recurrent falls & 1 & - & - & - & $1 / 1(100 \%)$ & 1 & - & Limited no \\
\hline Typical antipsychotics & Falls & 1 & - & - & - & $1 / 1(100 \%)$ & 1 & - & Limited no \\
\hline Risperidone & Falls & 1 & - & - & - & $1 / 1(100 \%)$ & 1 & - & Limited no \\
\hline Olanzapine & Falls & 1 & $1 / 1(100 \%)$ & 1 & - & - & - & - & Limited yes \\
\hline Antidepressant & Falls & 12 & $10 / 12(83 \%)$ & 10 & - & $2 / 12(17 \%)$ & 2 & - & Strong yes \\
\hline \multirow{2}{*}{$\begin{array}{l}\text { Tricyclic } \\
\text { antidepressant }\end{array}$} & Falls & 2 & $2 / 2(100 \%)$ & 2 & - & - & - & - & Strong yes \\
\hline & Recurrent falls & 1 & $1 / 1(100 \%)$ & 1 & - & - & - & - & Limited yes \\
\hline \multirow[t]{2}{*}{ SSRI } & Falls & 2 & $2 / 2(100 \%)$ & 2 & - & - & - & - & Strong yes \\
\hline & Injurious falls & 1 & $1 / 1(100 \%)$ & 1 & - & - & - & - & Limited yes \\
\hline Non-SSRI & Falls & 1 & - & - & - & $1 / 1(100 \%)$ & 1 & - & Limited no \\
\hline Trazodone & Falls & 1 & $1 / 1(100 \%)$ & 1 & - & - & - & - & Limited yes \\
\hline \multirow{2}{*}{$\begin{array}{l}\text { Multiple psychotropic } \\
\text { drugs }\end{array}$} & Falls & 3 & $3 / 3(100 \%)$ & 3 & - & - & - & - & Strong yes \\
\hline & Recurrent falls & 1 & $1 / 1(100 \%)$ & 1 & - & - & - & - & Limited yes \\
\hline
\end{tabular}

Positive findings: the association between the use of psychoactive drugs and falls is strong (OR, RR or $\mathrm{HRR}>2.0$ or $<0.5$ or significant, $\mathrm{p}<0.05)$. 
et al., 2003). Positive findings for the risk of recurrent falls were found in one out of two (50\%) cohorts (Thapa et al., 1995). However, when we examined these drugs separately, the evidence for the individual drug classes differed from the overall evidence. Based on only one cohort, we found limited evidence that benzodiazepines increase the risk of recurrent falls (Thapa et al., 1995), and that intermediate- and long-acting benzodiazepines increase overall fall risk. We also found limited evidence that short-acting benzodiazepines increase fall risk at night-time but not during the day (Ray et al., 2000). For the whole spectrum of benzodiazepines, the individual effects described above disappear.

Furthermore, we found strong evidence that the use of anti-anxiety drugs increases fall risk. Positive findings were found in two out of two studies $(\mathrm{n}=2015$, RR 1.32 and $\mathrm{n}=18,855$, OR 1.22) (van Doorn et al., 2003; Kiely et al., 1998).

We found inconclusive evidence for the use of sedatives. Positive findings were found in only one out of four studies (Lord et al., 2003).

There is strong evidence that the use of hypnotics does not increase fall risk. In the two studies we included, there were no significant associations found between the use of hypnotics and (recurrent) falls $(n=34,163$, OR $1.13(0.98$ 1.30) and $\mathrm{n}=282, \mathrm{IDR}=1.23$ (0.55-2.76) (Avidan et al., 2005 and Thapa et al., 1995, respectively).

\section{ANTIPSYCHOTICS}

The evidence that antipsychotics increase fall risk is inconclusive. Positive findings were found in three out of seven (43\%) cohorts (Kiely et al., 1998; van Doorn et al., 2003; Hien et al., 2005). However, after stratification by type of antipsychotic, there is limited evidence that olanzapine use increases fall risk, and limited evidence that risperidone and typical antipsychotics do not increase fall risk. There is limited evidence that antipsychotics do not increase the risk of recurrent falls (Hien et al., 2005).

\section{ANTIDEPRESSANTS}

There is strong evidence that the use of antidepressants increases fall risk. In 10 out of $12(83 \%)$ cohorts significant associations were found $(n=78-2428$, range of effect sizes 1.1-7.6) (Lipsitz et al., 1991; Thapa et al., 1995; 1998; Arfken et al., 2001; van Doorn et al., 2003; Lord et al., 2003; Rosendahl et al., 2003; Hien et al., 2005). After stratification by the categories of antidepressants, the evidence that the use of tricyclic antidepressants $(2 / 2$ cohorts, $n=282$, IDR 2.96 and $\mathrm{n}=2428, \mathrm{RR} 2.0$ ) (Thapa et al., 1995; 1998) and the use of SSRIs ( $2 / 2$ cohorts, $\mathrm{n}=368$, OR 2.01 and $\mathrm{n}=2428, \mathrm{RR} 1.8$ ) (Arfken et al., 2001; Thapa et al., 1998) increase fall risk remains strong. The evidence that the use of trazodone increases fall risk is limited (Thapa et al., 1998).

\section{MULTiPle PSYCHOTROPIC DRUGS}

There is strong evidence that multiple psychoactive drug use increases fall risk (3/3 studies, $n=177-282$, range of $R R$ 1.30-10.3) (Thapa et al., 1995; Lord et al., 2003; Cooper et al., 2007). One study classified multiple drugs as the use of 
two psychotropics (RR 3.2), three psychotropics (RR 6.7) or four psychotropics (RR 10.3) (Cooper et al., 2007). The evidence for recurrent falls is limited (Thapa et al., 1995).

\section{Discussion}

This systematic review has summarized the results of 17 prospective cohort studies concerning the influence of psychoactive drug use on fall risk and the influence of these drugs on gait parameters in nursing home populations with residents who have dementia. Substantial heterogeneity across studies for determinant measures, outcome measures, statistical analysis and data presentation was found. This heterogeneity impeded sensible statistical pooling of results; hence, a qualitative summary was undertaken. Strong evidence was found for the use of multiple psychotropic drugs, antidepressants and antianxiety drugs to increase fall risk. Strong evidence was found that hypnotics did not increase fall risk. The reported strength of the associations varied widely in the evidence for multiple psychotropic drugs (RR 1.30-10.3). The strength of the significant association seems to be moderate in one study $(\mathrm{RR}=1.30)$ (Lord et al., 2003), whereas in another study the strength is larger $(R R=10.3)$ for the concurrent use of four psychotropics (Cooper et al., 2007). The evidence was based on three smaller cohorts (Thapa et al., 1995; Lord et al., 2003; Cooper et al., 2007). The conclusion of strong evidence that the use of anti-anxiety drugs increases fall risk is based on only two cohorts. Although the strength of the associations in these two cohorts is moderate (RR 1.32, OR 1.22), the two cohorts were large (Kiely et al., 1998; van Doorn et al., 2003). The strong evidence for the use of antidepressants is based on 10 cohorts (Lipsitz et al., 1991; Thapa et al., 1995; 1998; Arfken et al., 2001; van Doorn et al., 2003; Lord et al., 2003; Rosendahl et al., 2003; Hien et al., 2005), with the strongest association ( $\mathrm{OR}=7.6)$ being found in a relatively small cohort ( $\mathrm{n}=126$ women) (Lipsitz et al., 1991). In the largest cohort $(\mathrm{n}=2428)$ only a weak association was found ( $R R=1.1)$ (Thapa et al., 1998).

For other drug classes, the evidence was limited or inconclusive. Limited evidence was always because the evidence was based on only one cohort.

It is generally recommended to prescribe benzodiazepines with a short elimination half-life to older persons. However, these were found to increase night-time falls (Thapa et al., 1995), which can be particularly hazardous. Intermediate- and long-acting benzodiazepines were found to increase overall fall risk (Thapa et al., 1995).

An earlier review on the association between psychoactive drugs and falls found an increased fall risk for all psychoactive drugs (Leipzig et al., 1999). However, the Leipzig review was performed in the general population, not exclusively in nursing home residents. Possible explanations for these inconsistent findings might lie with our different methodology and review criteria, and with our qualitative summary using levels of evidence. We only included papers with a prospective study design because this is considered to be the optimal design to identify the presence of prognostic factors and their 
associations with the outcome (Altman, 2001). The Leipzig review also included studies with a cross-sectional and a case control study design (Leipzig et al., 1999).

\section{Limitations of this review}

The lack of homogeneity across the studies impeded sensible statistical pooling of data. This is a limitation of our study, as we had to define levels of evidence based on the strength of positive and negative findings across the studies for each medication type or group, and each outcome. A particular limitation of this approach is that the strength of findings is not strengthened or ameliorated depending upon the sample size, which is an important influence when pooled data is incorporated into a meta-analysis. A large study with a moderate positive effect contributes substantially more to a pooled effect size than does a small sample study with the same positive effect.

The fact that none of the studies we included presented a sub-group analysis of the estimates in the population of nursing home residents with dementia could have biased our conclusions. The exact contribution of psychoactive drug use to fall risk in nursing home residents with dementia is not yet known.

Also, the presentation of the different drug classes in the papers could have biased our conclusions. Some papers classified all psychoactive drugs together, regardless of specific drug class. Furthermore, the difference between antianxiety, sedative and hypnotic characteristics of psychoactive drugs is often a matter of dose and of elimination half-life. In general, benzodiazepines are prescribed as a hypnotic, anti-anxiety drug or sedative. The overall level of evidence for benzodiazepines is inconclusive, which may be due to the fact that there is strong evidence that anti-anxiety drugs increase fall risk and limited evidence that intermediate- and long-acting benzodiazepines increase fall risk, and that there is strong evidence that hypnotics do not increase fall risk and limited evidence that short-acting benzodiazepines do not increase fall risk.

Levels of evidence in this review were based on positive findings from multivariate or univariate results. The use of univariate results when multivariate results were not available could have biased our conclusions regarding the level of available evidence. Overestimation of the estimates may occur because univariate results are not adjusted for potential confounding.

The possibility of publication bias cannot be excluded. One cohort published three articles (Thapa et al., 1998; Ray et al., 2000; 2002). Studies with significant results are more likely to lead to multiple publications. Furthermore, relevant studies hidden in unknown databases are difficult to locate and therefore may have been missed.

\section{Validity of the studies in the review}

Information bias can result from differential and non-differential misclassification and can influence the estimate of the strength of the association. The incidence reports and medical records from which falls were ascertained may not be complete. On the other hand, a recorded fall may not be a fall according 
to the definition, as acute medical conditions may have been involved in the population under study (Kellogg International Work Group, 1987).

Misclassification of drug use may result when drug use is ascertained only from medical records and when it is not assured that medications were actually administered. Baseline measurement of drug use can induce substantial misclassification. One study found that this misclassification caused substantial underestimation of the association of benzodiazepine use with fall risk (Ray et al., 2002).

Finally, selective loss to follow-up cannot be excluded in all studies. In one cohort, residents were followed through the day of facility exit, defined as discharge, death or transfer or a hospital stay of more than 14 days (Thapa et al., 1998; Ray et al., 2000; 2002).

\section{Conclusions and recommendations}

In summary, we conclude that the studies conducted within the period covered by this review consistently show an increased fall risk for the use of multiple drugs, antidepressants and anti-anxiety drugs in nursing home populations with residents with dementia. The evidence for other psychoactive drug classes is limited or inconclusive. Our initial approach was to analyze the data of nursing home residents with dementia only. However, none of the studies we found used a sub-group analysis for this specific group of residents.

It is generally accepted that falls are an intrinsic component of dementia and living in a nursing home. However, because of the multi-morbidity of this patient group, we do not know which risk factors are (potentially) reversible. The relative contribution of each drug class is not clear from the current literature. Also, little is known about dose and duration of use in relation to fall risk.

It was revealing to discover how little is known about the influence of psychoactive drugs on gait parameters in nursing home residents with dementia. As drug withdrawal has been shown to reduce fall risk (Campbell et al., 1999) and improve mobility tests in community-dwelling older persons without dementia (van der Velde et al., 2007a; 2007b), it is important to know the effect of psychoactive drugs on gait in nursing home residents with dementia. Falls due to psychoactive drug use might be caused by impairment of mobility generated by these drugs (Lord et al., 1995). If gait can be improved by withdrawal of these drugs, a number of falls might be prevented, even among nursing home residents. Gait measurements may be useful in the clinical follow-up of fallers in whom these drugs are withdrawn. Large prospective studies on the relationship between psychoactive drugs and gait in nursing home residents with dementia are needed, and should focus on the contribution of each drug class and dose and duration of use on fall risk.

\section{Conflict of interest}

None. 


\section{Description of authors' roles}

Carolyn Sterke, Arianne Verhagen and Tischa van der Cammen were responsible for the design of the study and performed the literature search. Carolyn Sterke and Tischa van der Cammen selected the articles and assessed their quality, and Carolyn Sterke extracted and analyzed the data and wrote the manuscript. Arianne Verhagen extracted the data and helped with the resolution of disagreements between reviewers. Ed van Beeck advised on the study and participated in the writing of the manuscript. Tischa van der Cammen initiated and developed the study, participated in and supervised the writing of the manuscript.

\section{References}

Altman, D. G. (2001). Systematic reviews of evaluations of prognostic variables. BMF, 323, 224-228.

Arfken, C. L., Wilson, J. G. and Aronson, S. M. (2001). Retrospective review of selective serotonin reuptake inhibitors and falling in older nursing home residents. International Psychogeriatrics, 13, 85-91.

Ariens, G. A. M., van Mechelen, W., Bongers, P. M., Bouter, L. M. and van der Wal, G. (2000). Physical risk factors for neck pain. Scandinavian fournal of Work Related Environment and Health, 26, 7-19.

Avidan, A. Y., Fries, B. E., James, M. L., Szafara, K. L., Wright, G. T. and Chervin, R. D. (2005). Insomnia and hypnotic use, recorded in the minimum data set, as predictors of falls and hip fractures in Michigan nursing homes. Fournal of the American Geriatrics Society, 53, 955-962.

Bueno-Cavanillas, A., Padilla-Ruiz, F., Jiménez-Moleón, J. J., Peinado-Alonso, C. A. and Gálvez-Vargas, R. (2001). Risk factors in falls among the elderly according to extrinsic and precipitating causes. European fournal of Epidemiology, 16, 849-859.

Capezuti, E., Evans, L., Strumpf, N. and Maislin, G. (1996). Physical restraint use and falls in nursing home residents. Fournal of the American Geriatrics Society, 44, 627-633.

Campbell, A. J., Robertson, M. C., Gardner, M. M., Norton, R. N. and Buchner, D. M. (1999). Psychotropic medication withdrawal and a home-based exercise program to prevent falls: a randomized, controlled trial. Fournal of the American Geriatrics Society, 47, 850-853.

Cooper, J. W., Freeman, M. H., Cook, C. L. and Burfield, A. H. (2007). Assessment of psychotropic and psychoactive drug loads and falls in nursing facility residents. The Consultant Pharmacist, 22, 483-489.

Dutch Cochrane Centre (n.d.). http://www.cochrane.nl/downloads.

Ensrud, K. E. et al. (2002). Central nervous system - active medications and risk for falls in older women. Fournal of the American Geriatrics Society, 50, 1629-1637.

Heinze, C., Hakfens, R. J. G. and Dassen, T. (2007). Falls in German in-patients and residents over 65 years of age. Fournal of Clinical Nursing, 16, 495-501.

Hien Le, T. T. et al. (2005). Atypical antipsychotic medications and risk of falls in residents of aged care facilities. Fournal of the American Geriatrics Society, 53, 1290-1295.

Jensen, J., Lundin-Olsson, L., Nyberg, L. and Gustafson, Y. (2002). Falls among frail older people in residential care. Scandinavian fournal of Public Health, 30, 54-61.

Kellogg International Work Group (1987). The prevention of falls in later life. A report of the Kellogg International Work Group on the prevention of falls by the elderly. Danish Medical Bulletin, 34, 1-24. 
Kiely, D. K., Kiel, D. P., Burrows, A. B. and Lipsitz, L. A. (1998). Identifying nursing home residents at risk for falling. Fournal of the American Geriatrics Society, 46, 551-555.

Krueger, P. D., Brazil, K. and Lohfeld, L. H. (2001). Risk factors for falls and injuries in a long-term care facility in Ontario. Canadian Fournal of Public Health, 92, 117-120.

Kuchynka, J., Kaser, L. and Wettstein, A. (2004). Can falls in nursing homes be avoided? Schweizerische Rundschau für Medizin Praxis, 93, 1503-1508.

Landis, J. R. and Koch, G. G. (1977). The measurement of observer agreement for categorical data. Biometrics, 33, 159-174.

Leipzig, R. M., Cummings, R. G. and Tinetti, M. E. (1999). Drugs and falls in older people: a systematic review and meta-analysis: I. Psychotropic drugs. Fournal of the American Geriatrics Society, 47, 30-39.

Lipsitz, L. A., Jonsson, P. V., Kelley, M. M. and Koestner, J. S. (1991). Causes and correlates of recurrent falls in ambulatory frail elderly. Fournal of Gerontology, 46, M114-M122.

Lord, S. R., Anstey, K. J., Williams, P. and Ward, J. A. (1995). Psychoactive medication use, sensory-motor function and falls in older women. British fournal of Clinical Pharmacology, 39, $227-234$.

Lord, S. R. et al. (2003). Differing risk factors for falls in nursing home and intermediate-care residents who can and cannot stand unaided. Fournal of the American Geriatrics Society, 51, 1645-1650.

Morris, J. C., Rubin, E. H., Morris, E. J. and Mandel, S. A. (1987). Senile dementia of the Alzheimer's type: an important risk factor for serious falls. Fournal of Gerontology, 42, $412-417$.

Morris, J. N. et al. (1990). Designing the national resident assessment instrument for nursing homes. Gerontologist, 30, 293-307.

Nakamura, T., Meguro, K. and Sasaki, H. (1996). Relationship between falls and stride length variability in senile dementia of the Alzheimer type. Gerontology, 42, 108-113.

Ray, W. A., Thapa, P. B. and Gideon, P. (2000). Benzodiazepines and the risk of falls in nursing home residents. Fournal of the American Geriatrics Society, 48, 682-685.

Ray, W. A., Thapa, P. B. and Gideon, P. (2002). Misclassification of current benzodiazepine exposure by use of a single baseline measurement and its effects upon studies of injuries. Pharmacoepidemiology and Drug Safety, 11, 663-669.

Rosendahl, E., Lundin-Olsson, L., Kallin, K., Jensen, J., Gustafson, Y. and Nyberg, L. (2003). Prediction of falls among older people in residential care facilities by the Downton index. Aging Clinical and Experimental Research, 15, 142-147.

Ruthazer, R. and Lipsitz, L. A. (1993). Antidepressants and falls among elderly people in long-term care. American fournal of Public Health, 83, 746-749.

Sackett, D. L., Straus, S. E., Richardson, W. S., Rosenberg, W. and Haynes, R. B. (2000). Evidence-based Medicine. How to Practice and Teach EBM. Edinburgh: Churchill Livingstone.

Speechley, M. and Tinetti, M. E. (1990). Assessment of risk and prevention of falls among elderly persons: role of the physiotherapist. Physiotherapy Canada, 4, 75-79.

Studenski, S. et al. (1994). Predicting falls: the role of mobility and nonphysical factors. fournal of the American Geriatrics Society, 42, 297-302.

Thapa, P. B., Gideon, P., Fought, R. L. and Ray, W. A. (1995). Psychotropic drugs and risk of recurrent falls in ambulatory nursing home residents. American fournal of Epidemiology, 142, 202-211.

Thapa, P. B., Brockman, K. G., Gideon, P., Fought, R. L. and Ray, W. A. (1996). Injurious falls in nonambulatory nursing home residents: a comparative study of circumstances, incidence, and risk factors. Fournal of the American Geriatrics Society, 44, 273-278.

Thapa, P. B., Gideon, P., Cost, T. W., Milam, A. B. and Ray, W. A. (1998). Antidepressants and the risk of falls among nursing home residents. New England fournal of Medicine, 339, $875-882$. 
Tinetti, M. E., Speechley, M. and Ginter, S. F. (1988). Risk factors for falls among elderly persons living in the community. New England fournal of Medicine, 26, 1701-1707.

van Doorn, C. et al. (2003). Dementia as a risk factor for falls and fall injuries among nursing home residents. Fournal of the American Geriatrics Society, 51, 1213-1218.

van der Velde, N., Stricker, B. H. Ch., Pols, H. A. P. and van der Cammen, T. J. M.

(2007a). Risk of falls after withdrawal of fall-risk-increasing drugs: a prospective cohort study. British fournal of Clinical Pharmacology, 63, 232-237.

van der Velde, N., Stricker, B. H. Ch., Pols, H. A. P. and van der Cammen, T. J. M. (2007b). Withdrawal of fall-risk-increasing drugs in older persons: effect on mobility test outcomes. Drugs and Aging, 24, 691-699.

van der Windt, D. A. W. M. et al. (2000). Occupational risk factors for shoulder pain: a systematic review. Occupational and Environmental Medicine, 57, 433-442. 